\title{
Autonomic dysfunction in post-COVID patients with and witfhout neurological symptoms: a prospective multidomain observational study
}

\author{
Alex Buoite Stella ${ }^{1} \cdot$ Giovanni Furlanis $^{1} \cdot$ Nicolò Arjuna Frezza $^{2} \cdot$ Romina Valentinotti $^{3} \cdot$ Milos Ajcevic $^{1,4}$. \\ Paolo Manganotti ${ }^{1}$ (D)
}

Received: 8 June 2021 / Revised: 21 July 2021 / Accepted: 26 July 2021 / Published online: 12 August 2021

(c) The Author(s) 2021

\begin{abstract}
The autonomic nervous system (ANS) can be affected by COVID-19, and dysautonomia may be a possible complication in post-COVID individuals. Orthostatic hypotension $(\mathrm{OH})$ and postural tachycardia syndrome (POTS) have been suggested to be common after SARS-CoV-2 infection, but other components of ANS function may be also impaired. The Composite Autonomic Symptom Scale 31 (COMPASS-31) questionnaire is a simple and validated tool to assess dysautonomic symptoms. The aim of the present study was to administer the COMPASS-31 questionnaire to a sample of post-COVID patients with and without neurological complaints. Participants were recruited among the post-COVID ambulatory services for follow-up evaluation between 4 weeks and 9 months from COVID-19 symptoms onset. Participants were asked to complete the COMPASS-31 questionnaire referring to the period after COVID-19 disease. Heart rate and blood pressure were manually taken during an active stand test for $\mathrm{OH}$ and POTS diagnosis. One-hundred and eighty participants were included in the analysis (70.6\% females, $51 \pm 13$ years), and $\mathrm{OH}$ was found in $13.8 \%$ of the subjects. Median COMPASS-31 score was 17.6 (6.9-31.4), with the most affected domains being orthostatic intolerance, sudomotor, gastrointestinal and pupillomotor dysfunction. A higher COMPASS-31 score was found in those with neurological symptoms $(p<0.01)$, due to more severe orthostatic intolerance symptoms $(p<0.01)$, although gastrointestinal $(p<0.01)$, urinary $(p<0.01)$, and pupillomotor $(p<0.01)$ domains were more represented in the non-neurological symptoms group. This study confirms the importance of monitoring ANS symptoms as a possible complication of COVID-19 disease that may persist in the post-acute period.
\end{abstract}

Keywords COVID-19 · Long-COVID · Autonomic dysfunction · COMPASS-31 · Orthostatic intolerance

Paolo Manganotti

pmanganotti@units.it

1 Clinical Unit of Neurology, Department of Medicine, Surgery and Health Sciences, Trieste University HospitalASUGI, University of Trieste, Strada di Fiume, 447, 34149 Trieste, Italy

2 School of Medicine and Surgery, Department of Medicine, Surgery and Health Sciences, Trieste University Hospital-ASUGI, University of Trieste, Strada di Fiume, 447, 34149 Trieste, Italy

3 Infectious Diseases, Trieste University Hospital-ASUGI, University of Trieste, Strada di Fiume, 447, 34149 Trieste, Italy

4 Department of Engineering and Architecture, University of Trieste, Via Alfonso Valerio 10, Trieste, Italy

\section{Introduction}

Novel coronavirus disease (COVID-19) has been suggested to not only affect health during the acute phase of the SARSCoV-2 infection, but some manifestations have been consistently reported in several studies also after the recovery. Such phenomenon has been named "long-COVID" or "post-acute COVID-19" depending on the time course definition of the persisting or novel symptoms [1], the latter being defined as a syndrome characterized by persistent symptoms and/or delayed or long-term complications beyond 4 weeks from the onset of COVID-19 [2]. These complications include myocarditis, pulmonary fibrosis, encephalitis, thromboembolic events, psychiatric illness, and persisting symptoms such as dyspnea, cough, and fatigue [3, 4]. Among these clinical manifestations, neurological symptoms may be also common, as cognitive deficits have been reported in $36 \%$ of patients two to four 
months after COVID-19 [5, 6], and the reported fatigue and dysexecutive syndrome may depend on cortical reorganization [7]. Despite few data are present and further investigations are recommended, age, higher BMI and female sex have been proposed as risk factors for post-COVID [8]. Some recent papers have pointed a rationale for autonomic dysfunction following COVID-19, suggesting a role of the virus infection and/or of the related immune response on the autonomic nervous system (ANS), often resulting in orthostatic intolerance (OI), including orthostatic hypotension $(\mathrm{OH})$ and postural tachycardia syndrome (POTS) [9, 10]. OH and POTS can be common in the elderly, particularly in geriatrics outpatients [11], as well as in individuals with other dysautonomic features like in neurological diseases (e.g., Parkinson or dementia) [12] or diabetes [13], the latter due to the possible presence of neuropathies affecting baroceptors and increased arterial stiffness [13, 14]. Such symptoms are often disabling and affect people's quality of life; additionally, they can increase the risk of further events and falls. A recent case series reported typical features of orthostatic intolerance, fatigue, and activity intolerance in post-COVID individuals, and autonomic testing found heterogeneous responses, including OH and POTS [15]. As of this time, reports of COVID-19-related dysautonomia have been limited, but some pathophysiological mechanisms have been suggested, as para- or post-infectious immune-mediated process [15], supported by the presence of dysautonomia in Guillain-Barré syndrome and acute autoimmune autonomic neuropathy $[16,17]$. For these reasons, patients with persisting or novel symptoms after COVID-19 should be assessed for autonomic disorders, including an active stand test measuring blood pressure and heart rate $[8,9]$ following standard procedures [18]. Due to the hypothesized influence of SARSCoV-2 infection on the autonomic nervous system, dysautonomia manifestations other than orthostatic syndrome might be supposed. The Composite Autonomic Symptom Scale 31 (COMPASS-31) questionnaire is a widely validated tool to assess symptoms of ANS dysfunction, and it evaluates six domains related to ANS function: OI, vasomotor, secretomotor, gastrointestinal, urinary, and pupillomotor [19].

The aim of this study was to assess the prevalence of ANS dysfunction, evaluated with the COMPASS-31 questionnaire and an active stand test, in a real-life setting in consecutive patients referred to the post-COVID ambulatory service, and to compare the patients who presented neurological symptoms with those without neurological manifestations.

\section{Materials and methods}

This prospective observational study included patients who referred to the post-COVID ambulatory service of the University Hospital and Health Services of Trieste (ASUGI) between the 15.02 and 15.05 of 2021 . All the procedures were performed according to the Declaration of Helsinki and the local institutional review board and ethics committee (CEUR-FVG) approved the study. To be included in the study, participants had to present persistent symptoms and/ or delayed or long-term complications between 4 weeks and 9 months from the onset of COVID-19 confirmed by a nasopharyngeal swab. Participants were excluded if before COVID-19 they suffered from ANS dysfunctions or cognitive impairment, or if they were prescribed medications that might alter ANS as: antidepressants, beta-blockers, ACE inhibitors, calcium channel blockers, alpha-1 blockers, antihistamines, cholinesterase inhibitors, and central antihypertensives. Those who reported a cognitive deficit as a post-COVID symptom received the Montreal Cognitive Assessment (MoCA) and neuropsychological evaluation, and if the corrected score was lower than 15 or the neuropsychological evaluation suggested a remarkable cognitive deficit, they were excluded from the analysis. Participants were grouped between those presenting post-COVID neurological symptoms based on the medical check performed by an infectious disease specialist working in the post-COVID ambulatory service and then confirmed by the neurologist according to the clinical and instrumental (where possible, e.g., nerve conduction study) evaluation.

\section{ANS dysfunction evaluation}

During the medical check, all the participants who met the inclusion and exclusion criteria were invited to complete the validated Italian version of the COMPASS-31 questionnaire [20]. To better assess post-COVID autonomic dysfunction, all questions were adapted to refer to the period after COVID-19, and improvements/worsening were evaluated comparing the time of the visit with the time when the dysautonomic symptoms appeared. A pilot testing was conducted on 10 post-COVID patients (not included in this study) on two separate occasions 14 days apart, according to previous surveys development [21-23] to investigate the reliability of the adapted version of the questionnaire employing a test-retest correlation that confirmed the appropriate reliability (Pearson's $r>0.900, p<0.01$ ). The total score of COMPASS-31 and domains score were computed according to the original instructions [19]. COMPASS-31 score was presented as a continuous variable (0-100) and a cutoff of 13.25 was used to suggest ANS dysfunction, as previously defined for diagnosis of multiple system atrophy with predominant parkinsonism [24]. Domains scores were presented as continuous variables; the maximum weighted scores for each subdomain are as follows: 40 for orthostatic intolerance, 5 for vasomotor dysfunction, 15 for secretomotor dysfunction, 25 for gastrointestinal (GI) dysfunction, 10 for urinary dysfunction, and 5 for pupillomotor dysfunction [19]. 
Although not present in the original COMPASS-31 questions, additional items were included for a better understanding of other possible features of ANS dysfunction following COVID-19. These questions included the subjective evaluation of (i) thermal/environmental comfort (patients were asked if after COVID-19 they felt more uncomfortable while staying in hot, cold, humid, or windy environments), (ii) heat and cold sensation (if they were more/less sensible to contact heat and cold), and (iii) sexual dysfunction (e.g., impotence and reduced lubrication). These items were scored with a yes/no answer.

The active stand test was performed at the end of the visit, following guidelines [18]. Measures were manually taken with a sphygmomanometer [25] by a trained neurologist in OI evaluation, asking the patient to rest in the supine position for $5 \mathrm{~min}$ before the first measure, and then $3 \mathrm{~min}$ after standing. $\mathrm{OH}$ was defined as a fall of $>20 \mathrm{mmHg}$ systolic and $>10 \mathrm{mmHg}$ diastolic after standing for $3 \mathrm{~min}$ [18], and corrected in case of supine hypertension [26], while POTS is characterized by orthostatic symptoms (in the absence of orthostatic hypotension) with an increase in heart rate of 30 beats per minute or more when standing for more than $30 \mathrm{~s}$ $[18,27]$.

\section{Statistical analysis}

All analyses were conducted using IBM SPSS Statistics (v.22.0, Chicago, IL, USA). Kolmogorov-Smirnov test of normality was performed. Categorical variables are presented as counts and percentages (\%), while normally distributed continuous variables are presented as means and standard deviations (SDs) and non-normally distributed continuous variables are presented as medians (25th-75th percentile). To determine whether the associations between disease features and severity and the total COMPASS-31 score resulted from global autonomic symptoms across multiple subdomains, we grouped patients by the number of positive subdomains (score $>0$ in any category) of the COMPASS-31: (1) the "global autonomic symptoms" group included all patients with five or more positive subdomains, and (2) the "limited autonomic symptoms" group included patients with fewer than five positive subdomains [28]. Post-COVID patients who presented neurological symptoms were compared to post-COVID patients without neurological symptoms, and further subgroup analysis compared the neurological patients according to their prevalent symptom. Between-groups comparisons were performed with the Mann-Whitney $U$ test and the Fisher's exact test. A univariate analysis was performed to investigate the association of different factors, including age, sex, and clinical characteristics, with the COMPASS-31 score. Factors that were associated with this score with $p<0.10$ were included in the stepwise multivariable model and were confirmed as significant if $p<0.05$, as previously described [29-31]. A binary logistic regression was performed between the COMPASS-31 total score and the OI domain, with the presence of $\mathrm{OH}$ and POTS as evaluated during the stand test. A value of $p<0.05$ was considered significant.

\section{Results}

\section{Demographics and clinical characteristics of the sample}

One-hundred and ninety-two patients were first included in data collection; after excluding those who had a remarkable cognitive impairment, 180 participants were included in the final analysis ( $70.6 \%$ females, $51 \pm 13$ years). Among these, 97 were characterized by neurological manifestations (myalgia/asthenia $22.7 \%$, headache $13.4 \%$, hyposmia/hypogeusia $37.1 \%$, dizziness $7.2 \%$, sleep disturbances $10.3 \%$, "brain fog"/ cognitive deficit $42.3 \%$ ), while 83 did not presented neurological symptoms but reported other post-COVID complications (61.3\% exertional dyspnea, $29.1 \%$ arthralgia, $9.6 \%$ other) (Table 1). The median time from COVID-19 onset to the visit was 59 (31-175 days).

\section{ANS dysfunction}

The median COMPASS-31 total score was $17.6(6.9-31.4)$ and was higher than 13.25 in $61.1 \%$ of the sample (Fig. 1). The OI domain score was $6.0(0.0-20.0)$ and was higher than 0 in $51.1 \%$ of the sample. The vasomotor domain score was $0.0(0.0-0.0)$ and was higher than 0 in $23.3 \%$ of the sample. The secretomotor domain score was $2.0(0.0-6.0)$ and was higher than 0 in $54.4 \%$ of the sample. The GI domain score was $4.0(1.5-7.0)$ and was higher than 0 in $79.4 \%$ of the sample. The urinary domain score was $0.0(0.0-1.1)$ and was higher than 0 in $35.6 \%$ of the sample. The pupillomotor domain score was $1.1(0.0-2.2)$ and was higher than 0 in $68.3 \%$ of the sample. "Global autonomic symptoms" were found in $26.1 \%$ of the sample. Reduced tolerance to environmental conditions was found for heat (22.2\%), cold (37.2\%), humidity (15.6\%) and wind (7.2\%). Subjectively altered contact thermal sensation was found for heat $(10.6 \%)$ and cold (11.7\%). Sexual impairment was reported by $14.4 \%$ of the sample. Active stand test suggested the prevalence of $\mathrm{OH}$ in $13.8 \%$ of the sample, while POTS was found in none of the subjects. In particular, those with $\mathrm{OH}$ were mainly characterized by decreased diastolic blood pressure after $3 \mathrm{~min}$ standing $(-15 \pm 3 \mathrm{mmHg})$.

Subgroups analysis between post-COVID patients with neurological symptoms and post-COVID patients without neurological symptoms (Table 1) suggested that, despite no significant differences in demographics or prevalence of 
Table 1 COMPASS-31 score, domains, and additional items in post-COVID patients with and without neurological symptoms

\begin{tabular}{|c|c|c|c|}
\hline & $\begin{array}{l}\text { Neuro } \\
n=97\end{array}$ & $\begin{array}{l}\text { Others } \\
n=83\end{array}$ & Significance \\
\hline Age (y) & $53 \pm 14$ & $49 \pm 11$ & 0.073 \\
\hline Females $[n(\%)]$ & $67(69.1)$ & $60(72.3)$ & 0.743 \\
\hline \multicolumn{4}{|l|}{ COMPASS-31 } \\
\hline Score $[0-100]$ & $20.7(5.4-32.2)$ & $14.2(8.2-30.3)$ & 0.002 \\
\hline$>13.25[n(\%)]$ & $55(56.7)$ & $45(55.4)$ & 0.654 \\
\hline \multicolumn{4}{|l|}{ Orthostatic intolerance } \\
\hline Score $[0-40]$ & $12.0(0.0-20.0)$ & $0.0(0.0-20.0)$ & $<0.001$ \\
\hline$>0[n(\%)]$ & $53(54.6)$ & $39(47.0)$ & 0.369 \\
\hline \multicolumn{4}{|l|}{ Vasomotor } \\
\hline Score $[0-5]$ & $0.0(0.0-0.0)$ & $0.0(0.0-0.0)$ & 0.226 \\
\hline$>0[n(\%)]$ & $22(22.7)$ & $20(24.1)$ & 0.860 \\
\hline \multicolumn{4}{|l|}{ Secretomotor } \\
\hline Score $[0-15]$ & $2.0(0.0-6.0)$ & $2.1(0.0-6.4)$ & 0.227 \\
\hline$>0[n(\%)]$ & $53(54.6)$ & $45(54.2)$ & 1.000 \\
\hline \multicolumn{4}{|l|}{ Gastrointestinal } \\
\hline Score [0-24] & $3.0(0.0-6.1)$ & $4.5(2.7-7.1)$ & $<0.001$ \\
\hline$>0[n(\%)]$ & $66(68.0)$ & $77(92.8)$ & $<0.001$ \\
\hline \multicolumn{4}{|l|}{ Urinary } \\
\hline Score $[0-10]$ & $0.0(0.0-1.0)$ & $0.0(0.0-1.1)$ & $<0.001$ \\
\hline$>0[n(\%)]$ & $26(26.8)$ & $38(45.8)$ & 0.012 \\
\hline \multicolumn{4}{|l|}{ Pupillomotor } \\
\hline Score $[0-6]$ & $1.0(0.0-2.0)$ & $1.3(0.3-2.3)$ & $<0.001$ \\
\hline$>0[n(\%)]$ & $59(60.8)$ & $64(77.1)$ & 0.024 \\
\hline Global autonomic symptoms $[n(\%)]$ & $23(23.7)$ & $24(28.9)$ & 0.496 \\
\hline \multicolumn{4}{|l|}{ Environmental intolerance $[n(\%)]$} \\
\hline Heat & $17(17.5)$ & $23(27.7)$ & 0.109 \\
\hline Cold & $33(34.0)$ & $34(41.0)$ & 0.356 \\
\hline Humid & $8(8.2)$ & $20(24.1)$ & 0.004 \\
\hline Wind & $4(4.1)$ & $9(10.8)$ & 0.093 \\
\hline \multicolumn{4}{|l|}{ Subj. thermal sensation $[n(\%)]$} \\
\hline Heat & $7(7.2)$ & $12(14.5)$ & 0.145 \\
\hline Cold & $5(5.2)$ & $16(19.3)$ & 0.005 \\
\hline Sexual impairment $[n(\%)]$ & $13(13.4)$ & $13(15.7)$ & 0.677 \\
\hline
\end{tabular}

Means \pm standard deviations, medians (25th-75th percentile) and proportions are reported
ANS dysfunction (COMPASS-31 > 13.25), the post-COVID patients with neurological symptoms reported a higher overall COMPASS-31 score $(p<0.01)$ and OI score $(p<0.01)$, and less GI $(p<0.01)$, urinary $(p<0.05)$ and pupillomotor scores $(p<0.05)$ (Fig. 2). Similarly, intolerance to humidity $(p<0.01)$ and subjective cold sensation $(p<0.01)$ were less prevalent in the neurological symptoms group.

\section{Factors associated with ANS dysfunction}

A trend for a negative association between age and COMPASS-31 score $>13.25$ was found at the multivariate analysis $(p<0.072)$, suggesting younger subjects might present more dysautonomic symptoms, while sex was found significantly associated with COMPASS-31, suggesting females have a higher probability to develop ANS dysfunction (OR 2.771, 95\% CI 1.091-7.035; $p=0.032$ ). Binary logistic regression failed to show any association between the OI domain and $\mathrm{OH}$ assessed at the active stand test $(p=0.262)$. At the univariate analysis, both subjective altered heat sensation (OR 3.936, 95\% CI 1.087-14.253; $p=0.39$ ) and cold sensation (OR 4.609, 95\% CI 1.289-16.477; $p=0.019$ ) were significantly associated with COMPASS-31 score $>13.25$. However, only cold sensation was significantly associated with the presence of positive responses in the OI domain (OR 4.007, 95\% CI 1.271-12.631; $p=0.018$ ). In the subgroup analysis among the patients with neurological symptoms at the visit, the regression analysis failed to show any specific 


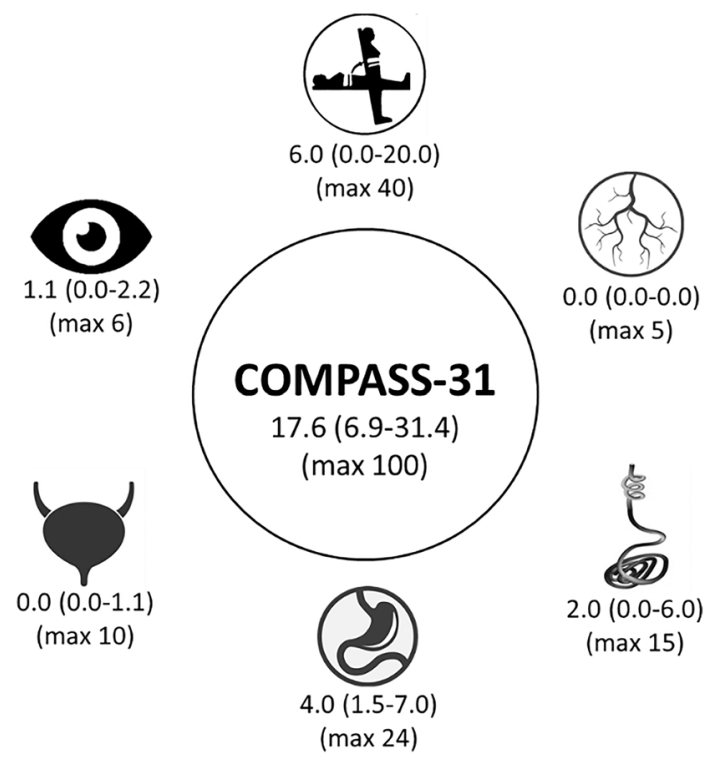

Fig. 1 Graphical representation of median (25th-75th percentile) COMPASS-31 total score and subdomains scores, with maximal score for each domain, in post-COVID individuals $(n=180)$. From top, clockwise: orthostatic intolerance, vasomotor, sudomotor, gastrointestinal, urinary, pupillomotor domains

association between the reported symptom(s) and ANS dysfunction or $\mathrm{OH}$, and non-significant difference was found in the OI subdomain score between those with and without $\mathrm{OH}$ at the active stand test.

\section{Discussion}

This study confirms and highlights the importance of assessing and monitoring ANS symptoms as possible long-term complications of SARS-CoV-2 infection. The main finding is the remarkable prevalence of ANS dysfunction in most postCOVID individuals, as shown by COMPASS-31 median and $>13.25$ score, independent of the presence or absence of neurological symptoms. The most affected domains were orthostatic, secretomotor, urinary, and pupillomotor impairment, and these findings are in line with preliminary reports using COMPASS-31 in post-COVID patients [32], with a prevalence of a "global dysautonomia" in about $1 / 4$ of the sample. Compared with previously published healthy controls from the literature [28, 33, 34], present data suggest a higher COMPASS-31 score in the post-COVID patients and ANS involvement. The absence of a significant association between age and COMPASS-31 score may depend on the specific questions asking for new dysautonomic symptoms after COVID-19, which might suggest an age-independent ANS response to the disease. Females were more commonly characterized by a higher COMPASS-31 score, in line with previous findings suggesting a higher prevalence of OI [35, 36] and symptomatic dysautonomia [37] in women. Comparison between patients with and without neurological complaining at the time of the visit suggested only minor differences, with a greater overall COMPASS-31 score in the neurological patients characterized by a higher OI domain score (despite no differences in the proportion of participants reporting at least one OI symptom) partially compensated by less GI, urinary and pupillomotor symptoms. Due to the absence of objective measures of autonomic function in the different domains, it is possible only to speculate that those with neurological symptoms post-COVID may suffer from more severe orthostatic impairment (despite some degree of lightheadedness may be present in all post-COVID participants), whereas those without neurological complications may be principally characterized by digestive and urinary alterations. The association between orthostatic impairment and neurological symptoms might be hypothesized due
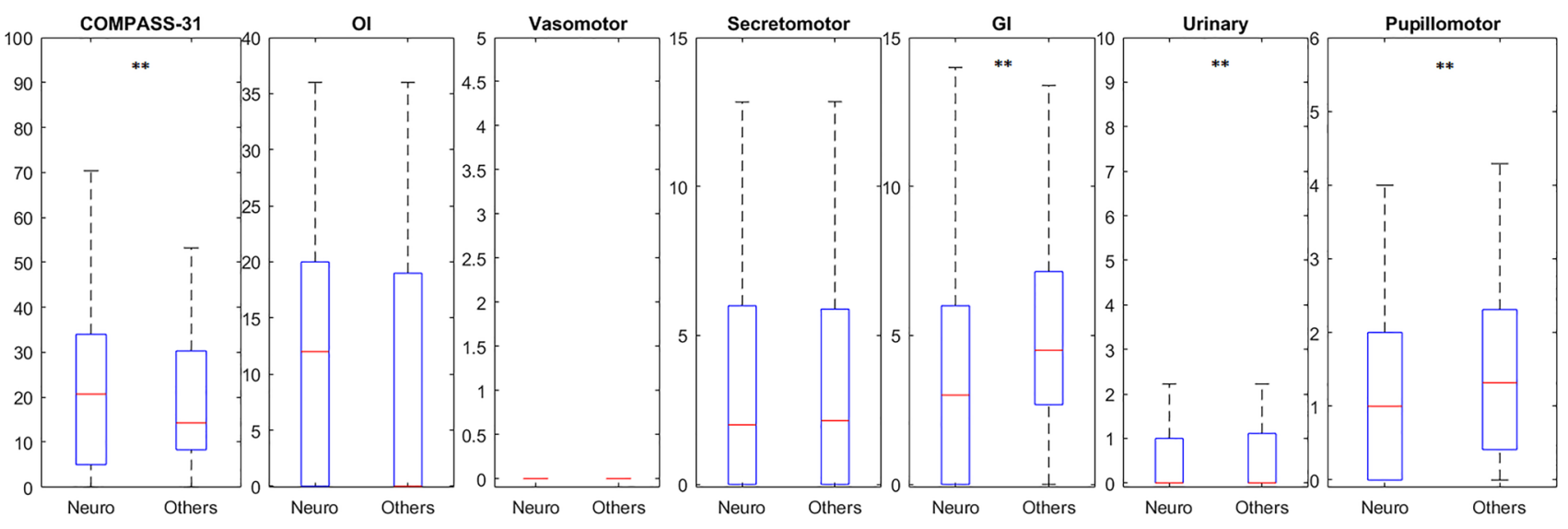

Fig. 2 Boxplots showing the comparison between patients with neurological symptoms (neuro) and patients without neurological symptoms (others) at the time of the visit. COMPASS-31 score and sub-

domains with corresponding maximal score: orthostatic intolerance (OI), vasomotor, secretomotor, gastrointestinal (GI), urinary, and pupillomotor. $* * p<0.01$ 
to the high prevalence of self-reported cognitive impairment that might be associated to altered postural vascular responses [38-40]. A common characteristic of the participants was the presence of fatigue, which can be a common post-COVID complication; however, no pathological differences between fatigued and non-fatigued patients on autonomic testing were previously found [41].

Despite few data have been published yet on dysautonomic symptoms and post-COVID, the rationale behind an ANS involvement during and after SARS-CoV-2 infection supports this study's observations [9, 10, 42, 43]. Some of the mechanisms that have been hypothesized to influence ANS in COVID-19 include the sympathetic activation inducing pro-inflammatory cytokines that are at the base of the cytokine response [44]. As such, overactivation of the sympathetic nervous system may underlie the development of some responses that have been suggested to influence COVID-19 symptoms and recovery. In addition, the immune response may influence ANS function [45], as previously reported for $\mathrm{OH}$ and POTS being associated with antibodies (e.g., $\alpha$-/ $\beta$-adrenoceptors and muscarinic receptors), previous infections and autoimmune disorders [46-48]. Despite none of the patients was diagnosed with POTS, and $\mathrm{OH}$ was present in about one individual on 10, about one-half of the sample reported at least one symptom, although mild, of OI. This discrepancy, and the absence of a significant association between the OI component of COMPASS-31 and the active stand test, are not surprising: indeed, blood pressure and heart rate measurements were manually taken at a conventional time of $3 \mathrm{~min}$, and this is known to potentially affect the assessment validity due to the measurement method and the different time courses of the responses [49]. Nevertheless, many questions still need to be clarified to understand the mechanisms underlying COVID-19 and vasovagal dysfunction, including the influence of SARSCoV-2 on cerebral blood flow, possible hypoxia secondary to residual lung damage, prolonged bed rest, and the development of neuropathies [10,50-55]. The present study included specific questions about subjective alterations in contact heat and cold sensations, which were reported to be altered in around $10 \%$ of the sample and were associated with higher COMPASS-31 total and OI domain scores. As such, these results support the hypothesis of a possible sensory or autonomic neuropathy involvement, although further studies including peripheral neurophysiology investigations are needed.

SARS-CoV-2 has been suggested to affect microcirculation, resulting in endothelial cell swelling and damage (endotheliitis), microscopic blood clots (microthrombosis), capillary congestion, and damage to pericytes, tissue repair angiogenesis, and scar formation [56]. In addition, a correlation is present between endothelial function and ANS [57], and vasomotor activity is a component of dysautonomia.
However, symptoms of the vasomotor domain were the less commonly reported alteration in this sample (about $1 / 4$ ), suggesting a minor impact of COVID-19 on vascular responses, or minor attention towards the related clinical manifestations (e.g., changes in hands and feet color).

About half of post-COVID individuals have reported secretomotor and sweating abnormalities. Together with the impaired environmental tolerance and thermal sensation, these observations suggest possible thermoregulatory alterations. However, since these findings were not supported by previously published objective measures (e.g., quantitative sensory testing-QST, or thermoregulatory responses in hot/ cold environments), it is not possible to confirm the hypothesis of impaired temperature regulation. Findings from a not yet published case report from our group found altered heat and cold sensation and pain in one patient who developed neuropathy after COVID-19. Heat, in combination with the COVID-19 pandemic, has been raised as a global concern due to the interference of cooling strategies and heat risk prevention strategies, with the recommendation to stay at home [58], and might be particularly relevant for the most vulnerable populations. Among these populations, people with cognitive disorders, reduced mobility, and autonomic dysfunction might be at a higher risk of heat risk [59]. Since most of these risk factors might be present in post-COVID patients, future actions should include this category of individuals among those at risk of heat-related illnesses.

GI symptoms can be present at COVID-19 onset, alone or in combination with other symptoms. Indeed, the angiotensin-converting enzyme 2 (ACE2) receptors, where the SARS-CoV-2 spike protein binds, is highly expressed in the gut [60], and viral invasion has been suspected due to the presence of the virus in the colonic tissue and feces [61, 62]. A previous study found the prevalence of GI symptoms during the post-COVID period in nearly half of the interviewed individuals [63]. Some of these sequelae, as abnormal appetite, nausea, and diarrhea, are also part of the GI domain of the COMPASS-31 score. However, the proportion of postCOVID patients who reported at least one GI symptom was far greater in our sample (more than 3/4).

Inflammation and demyelination of the pudendal nerve are associated with the possible development of bladder incontinence and urinary symptoms, with pudendal neuropathy being a possible feature of COVID-19 as also reported in previous viral infections in animals and humans [64, 65]. In the acute phase, increased urinary frequency has been previously reported in $1 / 8$ of male patients, and suggested to be secondary to viral cystitis due to underlying COVID-19 disease [66]. However, despite these potential mechanisms, a large cohort study found bladder dysfunction not significantly increased in the 2-5 months after COVID-19 infection [67]. In our sample, $1 / 4$ to $1 / 2$ of the sample reported some sort of urinary dysfunction, including both involuntary 
voids and voiding difficulty. Sexual impairment, in particular erectile dysfunction, has been suggested to be possible after COVID-19 due to both endothelial and autonomic components of male erection $[68,69]$. In addition, anosmia and ageusia, possible post-COVID complications, may participate in impaired sexual responses [70]. Nevertheless, it is not possible to reconduct sexual impairment only to ANS dysfunction, as psychogenic might play a fundamental role during a pandemic [71]. Our sample was characterized by similar proportions of reported sexual impairment in males and females; nevertheless, this is not surprising as sexual dysfunction in females was reported after COVID-19 disease [72].

Vision abnormalities have been reported in clinical practice by several COVID-19 and post-COVID individuals, including sore eyes and light sensitivity [73, 74]. Despite the possible influence of acute SARS-CoV-2 infection and hospitalization on ocular manifestations described in this sample, none of the participants reported eye complaints (e.g., acute conjunctivitis) during the acute phase of the disease, and an ANS involvement might be suspected. Indeed, pupil responses assessed with an automatic pupillometer were found impaired in patients recovering from COVID-19 [75]. Although this impairment might be present already during the acute phase in critically ill COVID-19 patients [76], controversial results have been found since ANS responses might be attenuated in the intensive care unit (ICU) population by ICU-related interventions, such as the administration of anesthetics, analgesics and inotropic medications that may have a prolonged effect [77]. In our sample, about one-half of the participants reported ocular impairments, and light sensitivity was the most common complaint.

This study has some limitations that need to be discussed. First, the primary ANS dysfunction evaluation was performed with a questionnaire that, despite a wide validation in different samples, suffers from possible subjective interpretation and emotional components. Indeed, the symptoms screened in the questionnaire and suggestive of ANS dysfunction are non-specific (e.g., diarrhea or urinary incontinence), and in absence of well-known condition with autonomic impairment characteristics, they might be a manifestation of disorders besides dysautonomia. Despite some studies are suggestive of an autonomic involvement after COVID-19 [9, 15], caution should be taken when considering COMPASS-31 results in this sample. The time reference for the symptoms was modified to adapt to the post-COVID period and did not affect the test-retest performance; nevertheless, some of the participants might have underrated some features that were already present before COVID-19 (e.g., GI or ocular symptoms). Second, the objective measure of OI was performed with a manual collection of heart rate and blood pressure during the active stand test, a procedure that is known to have a limited validity. Although continuous beat-to-beat monitoring should be preferred, such devices are still not common in many hospital and ambulatory services, and this data collection was performed during the first period of a dedicated ambulatory service for postCOVID manifestations. The authors are aware that objective and standard protocols for dysautonomic symptoms should be recommended; however, ANS dysfunction is often overlooked and patients are referred to dedicated centers only when persisting and severe symptoms are present. Even mild ANS impairment can be disabling and influence individuals' quality of life.

Unfortunately, we were not able to further investigate the reported symptoms with objective techniques due to the absence of local dedicated services with the appropriate devices and protocols, and patients were referred to national centers for dysautonomic disorders. At this time, we are not able to provide findings about the diagnostic accuracy of our screening due to the absence of further follow-up and a possible selection bias (only some individuals might have had the opportunity to reach the dysautonomia center for instrumental investigations). However, although possibly overestimated, this study provides evidence of self-reported common dysautonomic manifestations in post-COVID individuals and recommends ANS screening and subsequent instrumental diagnosis as part of follow-up monitoring.

\section{Conclusions}

This study conducted in post-COVID individuals with and without neurological manifestations revealed that most of the sample was characterized by dysautonomic symptoms, as reported with the COMPASS-31 score. Orthostatic intolerance, sudomotor, gastrointestinal, and pupillomotor abnormalities were commonly reported as complications of COVID-19. After an active stand test, about $10 \%$ of the individuals were characterized by a fall in blood pressure suggestive of orthostatic hypotension. Other dysfunctions include reduced tolerance to environmental conditions and sexual impairments. Taken together, these findings confirm the hypothesis of an ANS involvement after COVID-19, and recommend further clinical and research evaluations in post-COVID individuals with and without neurological symptoms.

Acknowledgements The authors want to thank the post-COVID ambulatory staff members and the participants. The authors thank Matteo di Franza for editorial assistance and proofreading. 
Funding Open access funding provided by Università degli Studi di Trieste within the CRUI-CARE Agreement. No funding was received for conducting this study.

\section{Declarations}

Conflicts of interest The authors have no conflicts of interest to declare.

Ethical approval All procedures performed in study were approved by the CEUR-FVG ethical committee.

Informed consent Informed consent was obtained from all individual participants included in the study.

Open Access This article is licensed under a Creative Commons Attribution 4.0 International License, which permits use, sharing, adaptation, distribution and reproduction in any medium or format, as long as you give appropriate credit to the original author(s) and the source, provide a link to the Creative Commons licence, and indicate if changes were made. The images or other third party material in this article are included in the article's Creative Commons licence, unless indicated otherwise in a credit line to the material. If material is not included in the article's Creative Commons licence and your intended use is not permitted by statutory regulation or exceeds the permitted use, you will need to obtain permission directly from the copyright holder. To view a copy of this licence, visit http://creativecommons.org/licenses/by/4.0/.

\section{References}

1. Alwan NA, Johnson L (2021) Defining long COVID: going back to the start. Med (New York, N.Y.) 2:501-504

2. Nalbandian A, Sehgal K, Gupta A et al (2021) Post-acute COVID19 syndrome. Nat Med 27:601-615. https://doi.org/10.1038/ s41591-021-01283-z

3. Cabrera Martimbianco AL, Pacheco RL, Bagattini ÂM, Riera R (2021) Frequency, signs and symptoms, and criteria adopted for long COVID: a systematic review. Int J Clin Pract. https://doi.org/ 10.1111/ijcp. 14357

4. Lund LC, Hallas J, Nielsen H et al (2021) Post-acute effects of SARS-CoV-2 infection in individuals not requiring hospital admission: a Danish population-based cohort study. Lancet Infect Dis. https://doi.org/10.1016/S1473-3099(21)00211-5

5. Blazhenets G, Schröter N, Bormann T et al (2021) Slow but evident recovery from neocortical dysfunction and cognitive impairment in a series of chronic COVID-19 patients. J Nucl Med. https://doi.org/10.2967/jnumed.121.262128

6. van den Borst B, Peters JB, Brink M et al (2020) Comprehensive health assessment three months after recovery from acute COVID19. Clin Infect Dis Off Publ Infect Dis Soc Am. https://doi.org/10. 1093/cid/ciaa1750

7. Versace V, Sebastianelli L, Ferrazzoli D et al (2021) Intracortical GABAergic dysfunction in patients with fatigue and dysexecutive syndrome after COVID-19. Clin Neurophysiol Off J Int Fed Clin Neurophysiol 132:1138-1143. https://doi.org/10.1016/j.clinph. 2021.03.001

8. Sudre CH, Murray B, Varsavsky T et al (2020) Attributes and predictors of Long-COVID: analysis of COVID cases and their symptoms collected by the Covid Symptoms Study App. medRxiv. https://doi.org/10.1101/2020.10.19.20214494
9. Dani M, Dirksen A, Taraborrelli P et al (2021) Autonomic dysfunction in "long COVID": rationale, physiology and management strategies. Clin Med 21:e63-e67. https://doi.org/10.7861/clinmed. 2020-0896

10. Raj SR, Arnold AC, Barboi A et al (2021) Long-COVID postural tachycardia syndrome: an American autonomic society statement. Clin Auton Res Off J Clin Auton Res Soc. https://doi.org/10.1007/ s10286-021-00798-2

11. Tran J, Hillebrand SL, Meskers CGM et al (2021) Prevalence of initial orthostatic hypotension in older adults: a systematic review and meta-analysis. Age Ageing. https://doi.org/10.1093/ageing/ afab090

12. Bengtsson-Lindberg M, Larsson V, Minthon L et al (2015) Lack of orthostatic symptoms in dementia patients with orthostatic hypotension. Clin Auton Res Off J Clin Auton Res Soc 25:87-94. https://doi.org/10.1007/s10286-014-0244-z

13. Kozakova M, Palombo C (2016) Diabetes mellitus, arterial wall, and cardiovascular risk assessment. Int $\mathbf{J}$ Environ Res Public Health 13:201. https://doi.org/10.3390/ijerph13020201

14. Takahashi M, Miyai N, Nagano S et al (2015) Orthostatic blood pressure changes and subclinical markers of atherosclerosis. Am J Hypertens 28:1134-1140. https://doi.org/10.1093/ajh/hpu301

15. Goodman BP, Khoury JA, Blair JE, Grill MF (2021) COVID-19 dysautonomia. Front Neurol 12:624968. https://doi.org/10.3389/ fneur.2021.624968

16. Chakraborty T, Kramer CL, Wijdicks EFM, Rabinstein AA (2020) Dysautonomia in Guillain-Barré syndrome: prevalence, clinical spectrum, and outcomes. Neurocrit Care 32:113-120. https://doi. org/10.1007/s12028-019-00781-w

17. Young RR, Asbury AK, Corbett JL, Adams RD (1975) Pure pandysautonomia with recovery. Description and discussion of diagnostic criteria. Brain 98:613-636. https://doi.org/10.1093/brain/ 98.4.613

18. Lahrmann H, Cortelli P, Hilz M et al (2006) EFNS guidelines on the diagnosis and management of orthostatic hypotension. Eur J Neurol 13:930-936. https://doi.org/10.1111/j.1468-1331.2006. 01512. $\mathrm{x}$

19. Sletten DM, Suarez GA, Low PA et al (2012) COMPASS 31: a refined and abbreviated composite autonomic symptom score. Mayo Clin Proc 87:1196-1201. https://doi.org/10.1016/j.mayocp. 2012.10.013

20. Pierangeli G, Turrini A, Giannini G et al (2015) Translation and linguistic validation of the composite autonomic symptom score COMPASS 31. Neurol Sci Off J Ital Neurol Soc Ital Soc Clin Neurophysiol 36:1897-1902. https://doi.org/10.1007/ s10072-015-2278-y

21. Buoite Stella A, Francescato MP, Sims ST, Morrison SA (2017) Fluid intake behavior in athletes during typical training bouts. J Sports Med Phys Fitness 57:1504-1512. https://doi.org/10.23736/ S0022-4707.16.06722-0

22. Buoite Stella A, Yardley J, Francescato MP, Morrison SA (2018) Fluid intake habits in type 1 diabetes individuals during typical training bouts. Ann Nutr Metab 73:10-18. https://doi.org/10.1159/ 000489823

23. Buoite Stella A, Ajčević M, Furlanis G et al (2020) Smart technology for physical activity and health assessment during COVID-19 lockdown. J Sports Med Phys Fitness. https://doi.org/10.23736/ S0022-4707.20.11373-2

24. Kim Y, Seok JM, Park J et al (2017) The composite autonomic symptom scale 31 is a useful screening tool for patients with Parkinsonism. PLoS One 12:e180744. https://doi.org/10.1371/journ al.pone. 0180744

25. McDonagh STJ, Mejzner N, Clark CE (2021) Prevalence of postural hypotension in primary, community and institutional care: a systematic review and meta-analysis. BMC Fam Pract 22:1. https://doi.org/10.1186/s12875-020-01313-8 
26. Metzler M, Duerr S, Granata R et al (2013) Neurogenic orthostatic hypotension: pathophysiology, evaluation, and management. J Neurol 260:2212-2219. https://doi.org/10.1007/ s00415-012-6736-7

27. Freeman R, Wieling W, Axelrod FB et al (2011) Consensus statement on the definition of orthostatic hypotension, neurally mediated syncope and the postural tachycardia syndrome. Clin Auton Res Off J Clin Auton Res Soc 21:69-72. https://doi.org/10.1007/ s10286-011-0119-5

28. Adler BL, Russell JW, Hummers LK, McMahan ZH (2018) Symptoms of autonomic dysfunction in systemic sclerosis assessed by the COMPASS-31 questionnaire. J Rheumatol 45:1145-1152. https://doi.org/10.3899/jrheum.170868

29. Furlanis G, Ajcevic M, Buoite Stella A et al (2020) Wake-up stroke: thrombolysis reduces ischemic lesion volume and neurological deficit. J Neurol 267:666-673. https://doi.org/10.1007/ s00415-019-09603-7

30. Buoite Stella A, Ajčević M, Furlanis G et al (2021) A physiological perspective of the associations between hydration status and CTP neuroimaging parameters in hyper-acute ischaemic stroke patients. Clin Physiol Funct Imaging. https://doi.org/10.1111/cpf. 12690

31. Ajcevic M, Furlanis G, Stella AB et al (2020) A CT perfusion based model predicts outcome in wake-up stroke patients treated with recombinant tissue plasminogen activator. Physiol Meas. https://doi.org/10.1088/1361-6579/ab9c70

32. Kedor C, Freitag H, Meyer-Arndt L et al (2021) Chronic COVID19 Syndrome and Chronic Fatigue Syndrome (ME/CFS) following the first pandemic wave in Germany-a first analysis of a prospective observational study. medRxiv. https://doi.org/10.1101/2021. 02.06 .21249256

33. Greco C, Di Gennaro F, D'Amato C et al (2017) Validation of the composite autonomic symptom score 31 (COMPASS 31 ) for the assessment of symptoms of autonomic neuropathy in people with diabetes. Diabet Med 34:834-838. https://doi.org/10.1111/dme. 13310

34. Treister R, O’Neil K, Downs HM, Oaklander AL (2015) Validation of the composite autonomic symptom scale 31 (COMPASS-31) in patients with and without small fiber polyneuropathy. Eur J Neurol 22:1124-1130. https://doi.org/10.1111/ene.12717

35. Anjum I, Sohail W, Hatipoglu B, Wilson R (2018) Postural orthostatic tachycardia syndrome and its unusual presenting complaints in women: a literature minireview. Cureus 10:e2435. https://doi. org/10.7759/cureus. 2435

36. Low PA, Sandroni P, Joyner M, Shen W-K (2009) Postural tachycardia syndrome (POTS). J Cardiovasc Electrophysiol 20:352358. https://doi.org/10.1111/j.1540-8167.2008.01407.x

37. Foschi M, Giannini G, Merli E et al (2021) Frequency and characteristics of dysautonomic symptoms in multiple sclerosis: a crosssectional double-center study with the validated italian version of the composite autonomic symptom score-31. Neurol Sci Off J Ital Neurol Soc Ital Soc Clin Neurophysiol 42:1395-1403. https://doi. org/10.1007/s 10072-020-04620-1

38. Novak P (2020) Post COVID-19 syndrome associated with orthostatic cerebral hypoperfusion syndrome, small fiber neuropathy and benefit of immunotherapy: a case report. eNeurologicalSci 21:100276

39. Miller AJ, Sheehan T, Bourne KM et al (2020) Attention and executive function are impaired during active standing in postural tachycardia syndrome. Auton Neurosci 227:102692. https://doi. org/10.1016/j.autneu.2020.102692

40. Wells R, Malik V, Brooks AG et al (2020) Cerebral blood flow and cognitive performance in postural tachycardia syndrome: insights from sustained cognitive stress test. J Am Heart Assoc 9:e017861. https://doi.org/10.1161/JAHA.120.017861
41. Townsend L, Moloney D, Finucane C et al (2021) Fatigue following COVID-19 infection is not associated with autonomic dysfunction. PLoS One 16:e0247280. https://doi.org/10.1371/journ al.pone. 0247280

42. Goldstein DS (2020) The extended autonomic system, dyshomeostasis, and COVID-19. Clin Auton Res Off J Clin Auton Res Soc 30:299-315. https://doi.org/10.1007/s10286-020-00714-0

43. Goldstein DS (2021) The possible association between COVID-19 and postural tachycardia syndrome. Hear Rhythm 18:508-509. https://doi.org/10.1016/j.hrthm.2020.12.007

44. Fudim M, Qadri YJ, Ghadimi K et al (2020) Implications for neuromodulation therapy to control inflammation and related organ dysfunction in COVID-19. J Cardiovasc Transl Res 13:894-899. https://doi.org/10.1007/s12265-020-10031-6

45. Guilmot A, Maldonado Slootjes S, Sellimi A et al (2021) Immune-mediated neurological syndromes in SARS-CoV-2-infected patients. J Neurol 268:751-757. https://doi.org/10.1007/ s00415-020-10108-x

46. Blitshteyn S, Brinth L, Hendrickson JE, Martinez-Lavin M (2018) Autonomic dysfunction and HPV immunization: an overview. Immunol Res 66:744-754. https://doi.org/10.1007/ s12026-018-9036-1

47. Ruzieh M, Batizy L, Dasa O et al (2017) The role of autoantibodies in the syndromes of orthostatic intolerance: a systematic review. Scand Cardiovasc J 51:243-247. https://doi.org/10.1080/ 14017431.2017.1355068

48. Watari M, Nakane S, Mukaino A et al (2018) Autoimmune postural orthostatic tachycardia syndrome. Ann Clin Transl Neurol 5:486-492. https://doi.org/10.1002/acn3.524

49. Breeuwsma AC, Hartog LC, Kamper AM et al (2018) Diagnosing orthostatic hypotension with continuous and interval blood pressure measurement devices. J Hum Hypertens 32:831-837. https:// doi.org/10.1038/s41371-018-0091-9

50. Manganotti P, Bellavita G, Tommasini V et al (2021) Cerebrospinal fluid and serum interleukins 6 and 8 during the acute and recovery phase in COVID-19 neuropathies patients. J Med Virol. https://doi.org/10.1002/jmv.27061

51. Buoite Stella A, Ajčević M, Furlanis G, Manganotti P (2021) Neurophysiological adaptations to spaceflight and simulated microgravity. Clin Neurophysiol 132:498-504. https://doi.org/ 10.1016/j.clinph.2020.11.033

52. Manganotti P, Bellavita G, D’Acunto L et al (2021) Clinical neurophysiology and cerebrospinal liquor analysis to detect GuillainBarré syndrome and polyneuritis cranialis in COVID-19 patients: a case series. J Med Virol 93:766-774. https://doi.org/10.1002/ jmv.26289

53. Chippa V, Aleem A, Anjum F (2021) Post acute coronavirus (COVID-19) syndrome. In: StatPearls. StatPearls Publishing, Treasure Island, FL

54. Su XW, Palka SV, Rao RR et al (2020) SARS-CoV-2-associated Guillain-Barré syndrome with dysautonomia. Muscle Nerve 62:E48-E49

55. Ghosh R, Roy D, Sengupta S, Benito-León J (2020) Autonomic dysfunction heralding acute motor axonal neuropathy in COVID-19. J Neurovirol 26:964-966. https://doi.org/10.1007/ s13365-020-00908-2

56. Østergaard L (2021) SARS CoV-2 related microvascular damage and symptoms during and after COVID-19: consequences of capillary transit-time changes, tissue hypoxia and inflammation. Physiol Rep 9:e14726. https://doi.org/10.14814/phy2.14726

57. Cieślik-Guerra UI, Fila M, Kamiński M et al (2014) Correlation between the activity of the autonomic nervous system and endothelial function in patients with acute coronary syndrome. Pol Arch Med Wewn 124:509-515. https://doi.org/10.20452/pamw. 2456 
58. Daanen H, Bose-O'Reilly S, Brearley M et al (2020) COVID-19 and thermoregulation-related problems: practical recommendations. Temp (Austin, Tex) 8:1-11. https://doi.org/10.1080/23328 940.2020.1790971

59. Buoite Stella A, Filingeri D, Ravanelli N et al (2021) Heat risk exacerbation potential for neurology patients during the COVID19 pandemic and related isolation. Int J Biometeorol 65:627-630. https://doi.org/10.1007/s00484-020-02044-2

60. Mao R, Qiu Y, He J-S et al (2020) Manifestations and prognosis of gastrointestinal and liver involvement in patients with COVID19: a systematic review and meta-analysis. Lancet Gastroenterol Hepatol 5:667-678. https://doi.org/10.1016/S2468-1253(20) 30126-6

61. Wu Y, Guo C, Tang L et al (2020) Prolonged presence of SARSCoV-2 viral RNA in faecal samples. Lancet Gastroenterol Hepatol $5: 434-435$

62. Xiao F, Tang M, Zheng X et al (2020) Evidence for gastrointestinal infection of SARS-CoV-2. Gastroenterology 158:1831-1833. e3. https://doi.org/10.1053/j.gastro.2020.02.055

63. Weng J, Li Y, Li J et al (2021) Gastrointestinal sequelae 90 days after discharge for COVID-19. Lancet Gastroenterol Hepatol 6:344-346

64. McMillan MT, Pan X-Q, Smith AL et al (2014) Coronavirusinduced demyelination of neural pathways triggers neurogenic bladder overactivity in a mouse model of multiple sclerosis. Am J Physiol Renal Physiol 307:F612-F622. https://doi.org/10.1152/ ajprenal.00151.2014

65. Pourfridoni M, Pajokh M, Seyedi F (2021) Bladder and bowel incontinence in COVID-19. J Med Virol 93:2609-2610

66. Mumm J-N, Osterman A, Ruzicka M et al (2020) Urinary frequency as a possibly overlooked symptom in COVID-19 patients: does SARS-CoV-2 cause viral cystitis? Eur Urol 78:624-628. https://doi.org/10.1016/j.eururo.2020.05.013

67. Welk B, Richard L, Braschi E, Averbeck MA (2021) Is coronavirus disease 2019 associated with indicators of long-term bladder dysfunction? Neurourol Urodyn. https://doi.org/10.1002/nau. 24682

68. Sansone A, Mollaioli D, Ciocca G et al (2021) Addressing male sexual and reproductive health in the wake of COVID-19 outbreak. J Endocrinol Invest 44:223-231. https://doi.org/10. 1007/s40618-020-01350-1

69. Sansone A, Mollaioli D, Ciocca G et al (2021) "Mask up to keep it up": preliminary evidence of the association between erectile dysfunction and COVID-19. Andrology. https://doi.org/10.1111/ andr.13003

70. Bertolo R, Cipriani C, Bove P (2021) Anosmia and ageusia: a piece of the puzzle in the etiology of COVID-19-related transitory erectile dysfunction. J Endocrinol Invest 44:1123-1124

71. Duran MB, Yildirim O, Kizilkan Y et al (2021) Variations in the number of patients presenting with andrological problems during the coronavirus disease 2019 pandemic and the possible reasons for these variations: a multicenter study. Sex Med 9:100292. https://doi.org/10.1016/j.esxm.2020.100292

72. Kaya Y, Kaya C, Tahta T et al (2021) Examination of the effect of COVID-19 on sexual dysfunction in women. Int J Clin Pract 75:e13923. https://doi.org/10.1111/ijcp.13923

73. Domínguez-Varela IA, Rodríguez-Gutiérrez LA, Morales-Mancillas NR et al (2021) COVID-19 and the eye: a review. Infect Dis (London, England) 53:399-403. https://doi.org/10.1080/23744 235.2021.1882697

74. Pardhan S, Vaughan M, Zhang J et al (2020) Sore eyes as the most significant ocular symptom experienced by people with COVID19: a comparison between pre-COVID-19 and during COVID-19 states. BMJ Open Ophthalmol 5:e00632. https://doi.org/10.1136/ bmjophth-2020-000632

75. Karahan M, Demirtaş AA, Hazar L et al (2021) Autonomic dysfunction detection by an automatic pupillometer as a non-invasive test in patients recovered from COVID-19. Graefe's Arch Clin Exp Ophthalmol: Albr von Graefes Arch fur Klin und Exp Ophthalmol 9:8-6. https://doi.org/10.1007/s00417-021-05209-w

76. Battaglini D, Santori G, Chandraptham K et al (2020) Neurological complications and noninvasive multimodal neuromonitoring in critically ill mechanically ventilated COVID-19 patients. Front Neurol 11:602114. https://doi.org/10.3389/fneur.2020.602114

77. Vrettou CS, Korompoki E, Sarri K et al (2020) Pupillometry in critically ill patients with COVID-19: a prospective study. Clin Auton Res Off J Clin Auton Res Soc 30:563-565 